\title{
Spontaneous bone regeneration in resected non-continuous mandible due to medication-related osteonecrosis of the jaw
}

\author{
Alparslan Esen ${ }^{1}$, Gokhan Gurses ${ }^{2}$, Sebnem Akkulah ${ }^{1}$ \\ ${ }^{1}$ Department of Oral and Maxillofacial Surgery, Faculty of Dentistry, Necmettin Erbakan University, \\ ${ }^{2}$ Department of Oral and Maxillofacial Surgery, Faculty of Dentistry, Selcuk University, Konya, Turkey
}

\begin{abstract}
J Korean Assoc Oral Maxillofac Surg 2021;47:465-470)
Few cases of spontaneous bone regeneration after extensive resection of the jaw bone have been reported, but it is more common in young adults or children. In this case, we report spontaneous bone healing in a 73-year-old female patient. On radiological examination, necrotic regions were seen in the right mandible. She was diagnosed with medication-related osteonecrosis of the jaw due to previous bisphosphonate use. After segmental resection, stabilization achieved using a reconstruction plate. The periosteum was preserved during the procedure. Twelve months later, panoramic radiography was taken and bone formation was seen both horizontally and vertically around the plate. If the periosteum is preserved and stabilization is achieved after resection in benign lesions, the bone may regenerate spontaneously regardless of age. Therefore, instead of simultaneous autogenous bone application, such patients may be followed to determine whether spontaneous bone healing will occur. This should improve patient comfort and reduce surgical cost.
\end{abstract}

Key words: Osteonecrosis, Bone regeneration, Periosteum, Mandible

[paper submitted 2020. 3. 16 / revised 2020. 5. 28 / accepted 2020. 6. 5]

\section{Introduction}

Bisphosphonates (BPs) are antiresorptive drugs used intravenously to treat cancer-related conditions, skeletal-related events associated with bone metastases such as breast, prostate and lung cancers, malignancy hypercalcemia, and lytic lesions caused by multiple myeloma. Furthermore, BPs are also used orally in osteoporosis and bone diseases such as osteopenia, Paget's disease, and osteogenesis imperfecta ${ }^{1}$. Hypotheses explaining the occurrence of medication-related osteonecrosis of jaws (MRONJ) are disruption of remodeling, inhibition of angiogenesis, excessive suppression of bone resorption, ongoing micro-trauma, soft tissue BP toxicity, inflammation, and infection ${ }^{2}$. In the literature, almost all cases

\footnotetext{
Alparslan Esen

Department of Oral and Maxillofacial Surgery, Faculty of Dentistry, Necmettin Erbakan University, Yaka Mh. Beysehir Cd. Baglarbasi Sk., Konya 42090, Turkey

TEL: +90-332-2200026

E-mail:alparslanesen@gmail.com

ORCID: https://orcid.org/0000-0001-7419-3210
}

(c) This is an open-access article distributed under the terms of the Creative Commons Attribution Non-Commercial License (http://creativecommons.org/ licenses/by-nc/4.0/), which permits unrestricted non-commercial use, distribution, and reproduction in any medium, provided the original work is properly cited.

Copyright (C) 2021 The Korean Association of Oral and Maxillofacial Surgeons. of spontaneous recovery after resection consist of young patients with benign lesions. Herein, we report a case of spontaneous regeneration of resected non-continuous mandible due to MRONJ in an elderly patient. There are two differences between this case and other cases of spontaneous bone formation after resection. Firstly, the patient had MRONJ that disrupted the repair mechanism of the bone and, secondly, the patient was old.

\section{Case Report}

A 73-year-old female patient presented to our clinic with an extraoral sinus tract in the posterior region of the right mandible.(Fig. 1) On intraoral examination, fistulas were seen with slight alveolar bone exposure on the lingual side. There were signs of inflammation around yellowish-colored exposed bone. The patient's history includes Parkinson's disease and mammary amputation due to breast cancer. There was no history of radiotherapy to the maxillofacial region. She underwent BP therapy ( $4 \mathrm{mg}$ zoledronate monthly) for 10 years. Also, there was no history of oral surgery or tooth extraction. The case was diagnosed as MRONJ Stage III based on the guidelines of the American Association of Oral and Maxillo- 


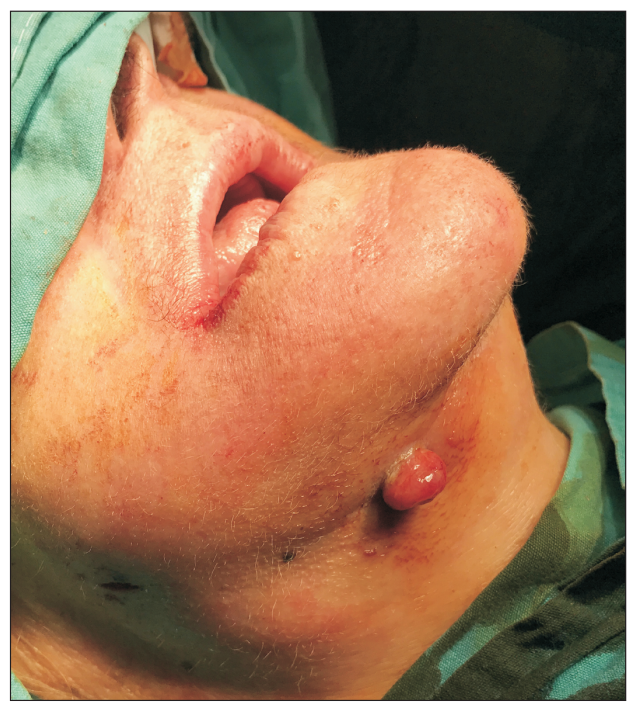

Fig. 1. An extraoral fistula originating from medication-related osteonecrosis of the jaw is seen in the submandibular region. Alparslan Esen et al: Spontaneous bone regeneration in resected non-continuous mandible due to medication-related osteonecrosis of the jaw. J Korean Assoc Oral Maxillofac Surg 2021

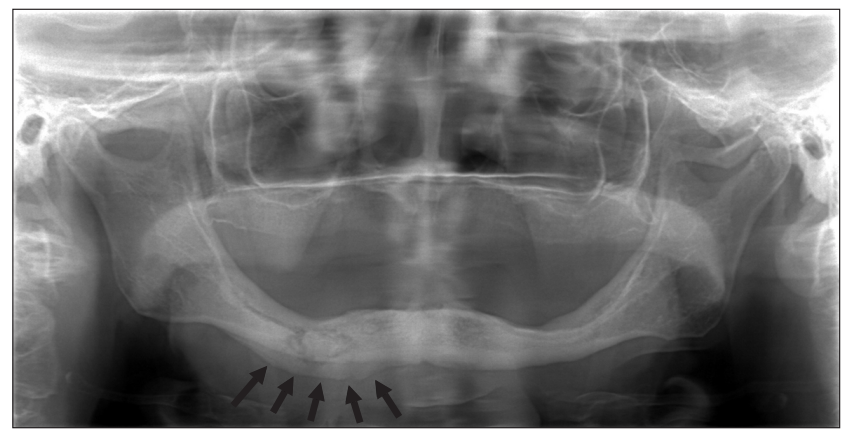

Fig. 2. The panoramic radiograph showed that about 3-cm-long lesion on the body of the mandible starting from the mental foramen and extending to the posterior region. At the same time, reactive bone formation was observed as a result of periosteal activation in the lower part of the lesion (arrows).

Alparslan Esen et al: Spontaneous bone regeneration in resected non-continuous mandible due to medication-related osteonecrosis of the jaw. J Korean Assoc Oral Maxillofac Surg 2021

facial Surgeons (AAOMS) ${ }^{1}$. Panoramic radiographs revealed a 3-cm-long lesion on the body of the mandible starting from the mental foramen and extending to the posterior region. The lesion also started from the alveolar crest and extended to include the lower border of the mandible. Radiographs also showed reactive bone formation as a result of periosteal activation in the lower part of the lesion.(Fig. 2) The reason for resection was extension of necrotic bone into the mandibular basis. After consulting with the oncologist, BP was discontinued. Oral antibiotics (amoxicillin+clavulanate $1 \mathrm{~g}$ ) and mouthwash (chlorhexidine digluconate) were used for

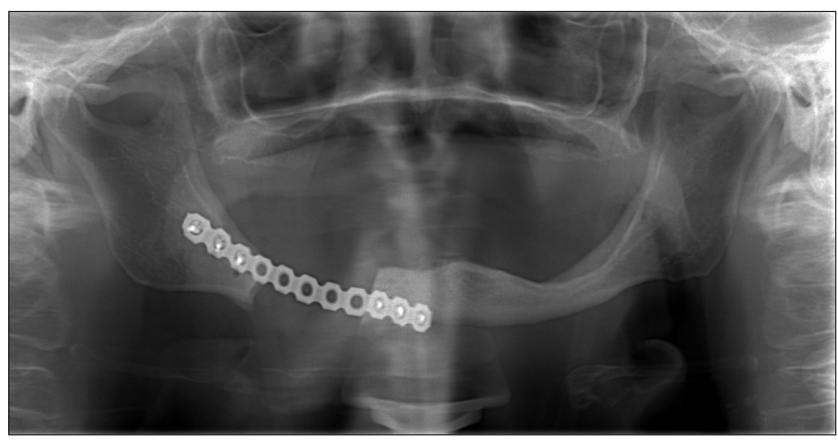

Fig. 3. The resection was performed and the reconstruction plate was fixed to the area.

Alparslan Esen et al: Spontaneous bone regeneration in resected non-continuous mandible due to medication-related osteonecrosis of the jaw. J Korean Assoc Oral Maxillofac Surg 2021

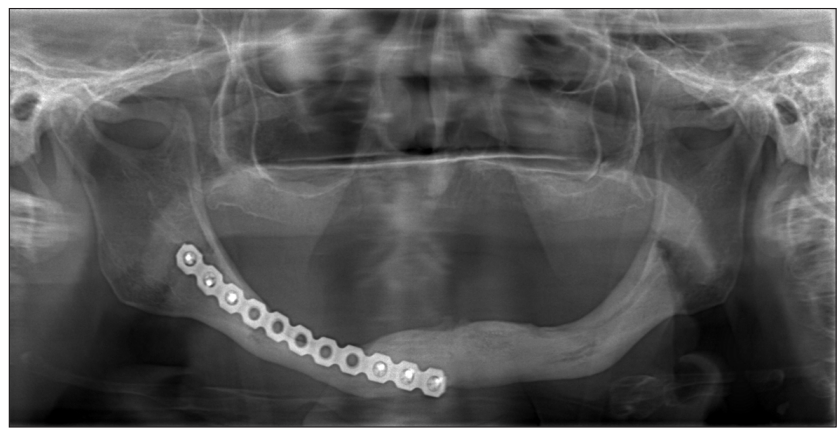

Fig. 4. One year later, complete bone regeneration was observed on the radiograph.

Alparslan Esen et al: Spontaneous bone regeneration in resected non-continuous mandible due to medication-related osteonecrosis of the jaw. J Korean Assoc Oral Maxillofac Surg 2021

10 days for the management of acute infection. After that the patient underwent partial mandibulectomy under general anesthesia. We used a subperiosteal incision in the intraoral approach. We elevated the mucoperiosteal flap without damaging the periosteum. To prevent displacement of the condyle, we first placed the reconstruction plate. Then, we removed the necrotic region via resection from healthy borders. The entire necrotic bone was removed with a safe margin of 5 $\mathrm{mm}$. During the surgical procedure, we tried to protect the periosteum. The extraoral fistula was completely removed and we performed wound revision in the region. After making sure that any necrotic bone residue in the area was completely removed, the surgical area was thoroughly washed with saline. The reconstruction plate was fixed and the intraoral flap was sutured for primary closure.(Fig. 3) We applied a tight horizontal matrix suture to prevent reopening of the wound and soft tissue collapse. The patient was prescribed oral antibiotics (amoxicillin+clavulanate $1 \mathrm{~g}$ ) and mouthwash (chlorhexidine digluconate) for 7 days postoperative. At the 
1-month follow-up visit, there were not any complications and healing of the extraoral sinus tract was observed. The 1-year follow-up panoramic radiographs showed complete regeneration of the bone.(Fig. 4)

\section{Discussion}

In the English literature, many case reports have described spontaneous recovery after mandibular segmental resection. All cases with spontaneous healing of the mandible consisted of benign lesions, and almost all occurred in young patients. Spontaneous recovery after MRONJ has not been reported in any of the cases published in the literature. The first case with spontaneous mandibular bone formation in the literature was a 15-year-old male patient who underwent resection due to ossifying fibroma. In this case, the periosteum was preserved and bone formation was first seen 3 months after the operation $^{3}$. In 1980, a 12-year-old male patient was reported to have a mandible defect because of a gunshot injury ${ }^{4}$. In this case, the fragments of bone remained and bone formation first seen 10 months after operation. In 1983, a case series of mandible resection due to various benign lesions in children was reported ${ }^{5}$. The ages ranged from 5 to 14 years and all had large defects. All patients had spontaneous bone formation 2 or 3 months after surgery ${ }^{5}$. In 1985, a case of severe avulsion of the mandible as a result of a war injury was reported ${ }^{6}$. Immobilization and stabilization of segments was achieved with Kirschner wire in a 7-year-old patient; 2.5 years postoperative, radiographs showed bone formation over the Kirschner wire. The author reported that the same technique was tried in older patients, but they did not achieve the same results. Therefore, he argued that the patient's age was a factor in spontaneous bone regeneration ${ }^{6}$.

Recently, 13 cases with spontaneous regeneration after mandibular segmental resections were reported by Anyanechi et al. ${ }^{7}$. In this retrospective study, the periosteum was completely excised in eight patients, and partially preserved in five patients, and those who underwent complete excision were younger. However, the soft tissue flap of the mandible was preserved in all cases. The authors thought that partial protection of the periosteum during segmental mandibular resection was more important in the development of spontaneous bone regeneration in an older age group than in a younger age group. However, they emphasized that early spontaneous bone regeneration occurred in the defect area even if the periosteum was not preserved in young patients. This unexpected spontaneous bone regeneration can be ex- plained by the mechanism of fracture healing, growth factors as a stimulus, and the soft tissue flap surrounding the defect area providing nutrients for undifferentiated mesenchymal cells to form new osteogenic tissue. In the same publication, intermaxillary fixation (IMF) for 6 weeks was the preferred stabilization technique?

Ruggiero and Donoff $f^{8}$ reported a case of spontaneous bone formation in irradiated bone. Even though there were cellular and vascular changes in the area where ionized radiation was applied, spontaneous regeneration was achieved by preserving the periosteum. Radiation thus does not completely eliminate the osteogenic potential of the periosteum. Nevertheless, it was emphasized that the bone formation capacity of periosteum was associated with dosing interval and total dose.

In the literature, it appears that there is no consensus concerning the mechanism of spontaneous bone regeneration in the mandible defect region. However, some influencing factors have been reported, such as patient age, protection of the periosteum as the source of osteogenic tissue, bone fragments providing osteogenic progenitor cells, local infection, and postoperative immobilization. Spontaneous bone regeneration can be evaluated in terms of fracture healing mechanism. Primary callus formation appearing as the initial reaction of the bone to an injury was previously described ${ }^{9}$. Rapid widespread cellular activity that involves the soft tissues around the defect mainly forms the external callus structure like a bridge to ensure the stability of the fragments. Later, remodeling continues to change from this temporary callus to mature bone formation. The periosteum is thought to be the primary source of osteogenic tissues, and contains two separate layers. The outer layer contains fibroblasts, blood vessels, and Sharpey's fibers, while the cambium layer (inner layer) consists of nerve fibers, capillaries, osteoblasts and undifferentiated mesenchymal stem cells. Undifferentiated mesenchymal cells in the periosteum support to the process of fracture healing via recapitulation of embryonic bone formation. In addition, growth factors in the inner layer are thought to play an important role in the formation of new bone and bone marrow by inducing mesenchymal cells in connective tissue $^{10}$.

In a very recent animal study in which segmental defects 3 $\mathrm{cm}$ in size were created in mini pigs, the researchers investigated the gene expression of the periosteum ${ }^{11}$. Gene expression and cell regeneration in this region was evaluated at 1 week and 2 weeks. The results showed that at 1 week, genes associated with inflammation and immune response were overexpressed. At week 2, genes associated with skeletal sys- 
tem development, bone development, regulation of ossification, and transforming growth factor-beta (TGF- $\beta$ ) and Wnt signaling were overexpressed. In other words, expression of important cytokines and proteins associated with ossification (TGF- $\beta$ and Bmp [bone morphogenetic proteins]) has been reported to occur as early as 2 weeks. The Bmp signaling pathway in periosteum plays a key role, especially in ossification. Thus, the role of the periosteum in spontaneous bone regeneration of the mandibular segmental defects was clearly revealed.

Recently, in an in vivo study on mice related to the role of the periosteum in bone regeneration, the periosteum was shown to contain skeletal stem cells and have a high degree of bone regeneration potential compared to bone marrow stromal cells (BMSCs). Researchers reported that although periosteal cells (PCs) and BMSCs are derived from a common embryonic mesenchymal lineage, PCs exhibit greater clonogenicity, growth and differentiation capacity than BMSCs. In the same study, it was concluded that PCs can contribute effectively to cartilage and bone repair ${ }^{12}$.

The soft tissue surrounding the defect region can also contribute significantly to bone formation. This soft tissue provides both the source of mesenchymal cells and the necessary blood supply for nutrition. The hematoma formed in the region contains angiogenic cytokine vascular endothelial growth factor (VEGF). In this way, angiogenesis can be induced and revascularization can be supported during bone repair $^{13}$. In cases where the periosteum is not intact, fragments from the periosteum, devitalized bone fragments in the surrounding tissue and remaining mandibular stumps (which also serve as a source of osteogenic cells) can be a source of bone regeneration ${ }^{4}$.

The histopathology of MRONJ is similar to osteomyelitis and osteoradionecrosis. The bone area affected by MRONJ is devoid of osteocytes, osteoblasts and normal bone marrow cells. Fibrosis has been reported in the bone marrow of the affected area, as in osteoradionecrosis. However, osteoradionecrosis shows a nonviable periosteum and does not exhibit reactive bone evidence, while MRONJ can occur with a viable periosteum and reactive bone ${ }^{14}$. Histopathological specimens taken from the resection margin in MRONJ cases revealed viable bone areas with inflamed vascular bone marrow spaces and numerous osteoclasts. There are both osteogenic and angiogenic precursor cells in the bone marrow. Cells in the bone marrow can produce factors such as BMP and VEGF that affect bone healing ${ }^{15}$. Therefore, it can be expected that vascular bone marrow areas in bone stumps after resection will contribute to bone regeneration through periosteal reactions.

Another factor suggested in the formation of spontaneous bone regeneration is the presence of infection. Chronic infection is thought to activate periosteal bone regeneration ${ }^{16}$. Other inflammatory lesions with bone formation include sclerosing osteomyelitis, condensing osteitis, and Garre's osteomyelitis. There is a chronic infection in all of these lesions. Therefore, it seems likely that the presence of infection will activate periosteal bone regeneration. In a rare case presented by Zhang et al. ${ }^{17}$, unexpected spontaneous bone regeneration was reported in a 48 -year-old patient after removal of vascularized fibular bone graft due to osteomyelitis. The authors stated that an adequate blood supply and osteogenic capacity were provided, particularly by preserving the periosteum of the fibular bone graft. In the same case, infection was also thought to activate the regeneration of the periosteal bone. In our case, when the patient first presented, she had an extraoral fistula with visible flow of pus, which the patient described as occurring for the prior 3 months. Preoperative panoramic radiography showed a reactive bone formation as a result of periosteal activation in the lower part of the lesion, as in Garre's osteomyelitis. Thus, we think that this chronic infection had an effect on periosteal activation in the formation of bone regeneration.

In our case, we applied preoperative antibiotic therapy to halt pus flow and lower postoperative infection risk. We did not encounter any infection in the postoperative period. The fistula region has also healed completely. We think that the intraoral approach and horizontal matrix suture application also affected this situation. Since the tissues were folded out with this suture technique, there was no collapse of the soft tissue in the defect region during the postoperative period.

IMF, Kirschner wires, reconstruction plates and external fixation devices are used for postoperative immobilization. Immobilization of bone fragments should be achieved in order for spontaneous bone regeneration to occur ${ }^{6,713}$. However, it has been reported that bone regeneration can occur without any stabilization ${ }^{18}$. In another retrospective study of 8 patients with a mean age of 10.75 years, complete spontaneous bone regeneration occurred in 6 patients and incomplete bone regeneration in 2 patients. In this study, the authors reported similar regenerative potential in two patients with incomplete bone regeneration without the use of stabilization devices (IMF or plates) $^{19}$. Although bone regeneration occurs without immobilization, complete regeneration and sufficient vertical bone regeneration do not occur. In this case, we had to 
ensure immobilization of the fragments, since it was a fully edentulous mandible. Therefore, there was an indication for a reconstruction plate to provide this fixation. Reconstruction plates are usually rigid, made of titanium and placed on the lower border of the mandible. This provides continuity of the facial contour and occlusion in bridge-shaped defects ${ }^{20}$. Various complications have been reported with reconstruction plates. These are infection, plate exposure, loosening or breaking of screws, and cracking of the plate ${ }^{20}$. In such cases, the plate should be removed. In addition, the patient feeling the plate through the soft tissue and discomfort related to temperature changes may also require the reconstruction plate to be removed. The timing of the removal of the plate varies according to the surgical procedure performed. These plates are applied in mandible fractures, distraction osteogenesis, and grafting processes after resection. Overall, radiography should show complete bone union and adequate radiopacity prior to plate removal. Reconstruction plates without bone grafting are used when the patient's overall health is compromised or when the patient does not accept a more extensive and more costly surgery. Otherwise, bone grafting and reconstruction plates can be used together. In our case, no complications occurred due to the reconstruction plate in the postoperative period. We did not want to remove the reconstruction plate with a second operation since the patient was elderly and had Parkinson's disease. Also, the patient did not want to undergo a second surgical procedure. We did not consider simultaneous grafting due to the possibility of a secondary infection in our patient due to MRONJ.

In the study of $\mathrm{Li}$ et al. ${ }^{11}, 6 \mathrm{~cm}$ was indicated as the critical defect size in order to display the osteogenesis potential of the periosteum ${ }^{9}$. In our case, a $3-\mathrm{cm}$ area was resected. Therefore, this size should be considered in order to obtain benefit from the activation of periosteal healing in segmental osteotomies. Otherwise, bone regeneration may not be sufficient to maintain bone continuity in the region. Vascularized bone grafts may be required for defects larger than this critical size.

We also believe that it is extremely important to preserve the periosteum for spontaneous bone regeneration, as evidenced by clinical results and experimental studies. Periosteal bone activation in young patients can be seen faster and ear$\operatorname{lier}^{5-7}$. However, as in our case and in another case report ${ }^{13}$, periosteal activation and bone regeneration may occur in an elderly patient, although it may take longer. An adequate blood supply is undoubtedly necessary for nutrition in the region. Therefore, we think it is also important to protect the soft tissue flap that covers the area and to ensure that it is tightly sutured. The vascular bone marrow, which may be located in bone stumps, can also contribute to bone formation with periosteal activation. In addition, immobilization of the remaining bone fragments may result in a smooth bone formation line. Normally, infection disrupts bone formation. However, as in osteomyelitis, we believe that the infection activated regeneration of the periosteal bone in our case. Preoperative radiography also showed periosteal bone activation under the lesion.

In conclusion, if the periosteum is preserved and stabilization is achieved after resection in benign lesions, the bone may regenerate spontaneously regardless of age. Therefore, instead of simultaneous autogenous bone application, monitoring to determine whether spontaneous bone healing occurs during follow-up is an alternative. This may improve patient comfort and reduce surgical cost. However, further studies on such cases and their outcomes are needed.

\section{ORCID}

Alparslan Esen, https://orcid.org/0000-0001-7419-3210

Gokhan Gurses, https://orcid.org/0000-0002-3825-4650

Sebnem Akkulah, https://orcid.org/0000-0001-5220-8119

\section{Authors' Contributions}

A.E. participated in data collection and wrote the manuscript. G.G. participated in the study design and performed the analysis of the references. S.A. participated in the study design and coordination and helped to draft the manuscript. All authors read and approved the final manuscript.

\section{Conflict of Interest}

No potential conflict of interest relevant to this article was reported.

\section{References}

1. Ruggiero SL, Dodson TB, Fantasia J, Goodday R, Aghaloo T, Mehrotra B, et al.; American Association of Oral and Maxillofacial Surgeons. American Association of Oral and Maxillofacial Surgeons position paper on medication-related osteonecrosis of the jaw--2014 update. J Oral Maxillofac Surg 2014;72:1938-56. https://doi.org/10.1016/j.joms.2014.04.031

2. Reid IR, Bolland MJ, Grey AB. Is bisphosphonate-associated osteonecrosis of the jaw caused by soft tissue toxicity? Bone 2007 ; 41:318-20. https://doi.org/10.1016/j.bone.2007.04.196

3. Kazanjian VH. Spontaneous regeneration of bone following exci- 
sion of section of the mandible. Am J Orthod Oral Surg 1946;32: 242-8. https://doi.org/10.1016/0096-6347(46)90016-6

4. Kisner WH. Spontaneous posttraumatic mandibular regeneration. Plast Reconstr Surg 1980;66:442-7.

5. Boyne PJ. The restoration of resected mandibles in children without the use of bone grafts. Head Neck Surg 1983;6:626-31. https:// doi.org/10.1002/hed.2890060203

6. Shuker S. Spontaneous regeneration of the mandible in a child. A sequel to partial avulsion as a result of a war injury. J Maxillofac Surg 1985;13:70-3. https://doi.org/10.1016/s0301-0503(85)800199

7. Anyanechi CE, Saheeb BD, Bassey GO. Spontaneous bone regeneration after segmental mandibular resection: a retrospective study of 13 cases. Int J Oral Maxillofac Surg 2016;45:1268-72. https:/ doi.org/10.1016/j.ijom.2016.04.011

8. Ruggiero SL, Donoff RB. Bone regeneration after mandibular resection: report of two cases. J Oral Maxillofac Surg 1991;49:64752. https://doi.org/10.1016/0278-2391(91)90349-q

9. McKibbin B. The biology of fracture healing in long bones. J Bone Joint Surg Br 1978;60-B:150-62. https://doi.org/10.1302/0301620X.60B2.350882

10. Einhorn TA. The cell and molecular biology of fracture healing. Clin Orthop Relat Res 1998;(355 Suppl):S7-21. https://doi. org/10.1097/00003086-199810001-00003

11. Li Z, Pan J, Ma J, Zhang Z, Bai Y. Microarray gene expression of periosteum in spontaneous bone regeneration of mandibular segmental defects. Sci Rep 2017;7:13535. https://doi.org/10.1038/ s41598-017-13586-8

12. Duchamp de Lageneste O, Julien A, Abou-Khalil R, Frangi G, Carvalho C, Cagnard N, et al. Periosteum contains skeletal stem cells with high bone regenerative potential controlled by Periostin. Nat Commun 2018;9:773. https://doi.org/10.1038/s41467-018-03124-Z

13. de Villa GH, Chen CT, Chen YR. Spontaneous bone regeneration of the mandible in an elderly patient: a case report and review of the literature. Chang Gung Med J 2003;26:363-9.

14. Marx RE, Tursun R. Suppurative osteomyelitis, bisphosphonate induced osteonecrosis, osteoradionecrosis: a blinded histopathologic comparison and its implications for the mechanism of each disease. Int J Oral Maxillofac Surg 2012;41:283-9. https://doi.org/10.1016/ j.ijom.2011.12.016

15. Qaisi M, Montague L. Bone margin analysis for osteonecrosis and osteomyelitis of the jaws. Oral Maxillofac Surg Clin North Am 2017;29:301-13. https://doi.org/10.1016/j.coms.2017.03.007

16. Elbeshir EI. Spontaneous regeneration of the mandibular bone following hemimandibulectomy. Br J Oral Maxillofac Surg 1990;28:128-30. https://doi.org/10.1016/0266-4356(90)90140-g

17. Zhang Z, Hu J, Ma J, Pan J. Spontaneous regeneration of bone after removal of a vascularised fibular bone graft from a mandibular segmental defect: a case report. Br J Oral Maxillofac Surg 2015;53: 650-1. https://doi.org/10.1016/j.bjoms.2015.04.002

18. Abdulai AE. Complete spontaneous bone regeneration following partial mandibulectomy. Ghana Med J 2012;46:174-7.

19. Okoturo E, Ogunbanjo OV, Arotiba GT. Spontaneous regeneration of the mandible: an institutional audit of regenerated bone and osteocompetent periosteum. J Oral Maxillofac Surg 2016;74:1660-7. https://doi.org/10.1016/j.joms.2016.02.007

20. Goh BT, Lee S, Tideman H, Stoelinga PJ. Mandibular reconstruction in adults: a review. Int J Oral Maxillofac Surg 2008;37:597605. https://doi.org/10.1016/j.ijom.2008.03.002

How to cite this article: Esen A, Gurses G, Akkulah S. Spontaneous bone regeneration in resected non-continuous mandible due to medication-related osteonecrosis of the jaw. J Korean Assoc Oral Maxillofac Surg 2021;47:465-470. https://doi. org/10.5125/jkaoms.2021.47.6.465 